\title{
Extraction of Citrus Flavonoids from Peel of Citrus Junos Using Supercritical Carbon Dioxide with Polar Solvent
}

\author{
Takuya Suetsugu $^{1,2}$, Hideo Iwai ${ }^{1,2}$, Masahiro Tanaka ${ }^{3}$, Munehiro Hoshino ${ }^{3}$, Armando Quitain ${ }^{1}$, Mitsuru Sasaki ${ }^{1}$, \\ Motonobu Goto ${ }^{4, *}$ \\ ${ }^{1}$ Graduate School of Science and Technology, Kumamoto University, Kurokami 2-39-1, Kumamoto, Japan \\ ${ }^{2}$ ASCII, Co Ltd, 2349 Tabara, Kawasaki-machi, Tagawagun, Fukuoka, Japan \\ ${ }^{3}$ Maruboshi Vinegar Co. Ltd., 2425 Tabara, Kawasaki-machi, Tagawagun, Fukuoka, Japan \\ ${ }^{4}$ Department of Chemical Engineering, Furo-cho, Chikusa-ku, Nagoya, Japan \\ *Corresponding author: mgoto@kumamoto-u.ac.jp
}

Received December 12, 2012; Revised November 02, 2013; Accepted December 15, 2013

\begin{abstract}
Citrus juice processing residues are mainly composed of peel, juice sack and seed. The peel, especially, consists of bioactive compounds such as flavones. Supercritical carbon dioxide $\left(\mathrm{SC}-\mathrm{CO}_{2}\right)$ extraction of flavonoid was carried out at a pressure of $30 \mathrm{MPa}$ and temperature ranging from 80 to $160^{\circ} \mathrm{C}$. Ratio of water and ethanol as a co-solvent was varied from 0 to $100 \%$. Flavonoids such as naringin and hesperidin have glycoside group, thus water was a good solvent for extraction of these compounds. Extraction behavior of tangeretin was quite different, the yield of tangeretin tended to increase with ethanol concentration.
\end{abstract}

Keywords: flavones, supercritical carbon dioxide, citrus junos

Cite This Article: Takuya Suetsugu, Hideo Iwai, Masahiro Tanaka, Munehiro Hoshino, Armando Quitain, Mitsuru Sasaki, and Motonobu Goto, "Extraction of Citrus Flavonoids from Peel of Citrus Junos Using Supercritical Carbon Dioxide with Polar Solvent." Chemical Engineering and Science 1, no. 4 (2013): 87-90. doi: 10.12691/ces-1-4-7.

\section{Introduction}

Most of Japanese sour citruses have been used for juice processing. One of them is Citrus junos, called yuzu, which is harvested mainly in Kochi Prefecture in Japan. Yuzu is a well-known citrus fruit in the southern part of Japan. Juice processing residue contains valuable compounds such as essential oil, pectin and flavonoids. The essential oil of yuzu is very expensive due to its unique characteristic odor and low yield. Recently, Hoshino et al. succeeded in effectively extracting essential oil from yuzu peel using a semi-continuous flow supercritical carbon dioxide $\left(\mathrm{SC}-\mathrm{CO}_{2}\right)$ extractor in our laboratory. $\mathrm{SC}-\mathrm{CO}_{2}$ extraction peel residue still contains

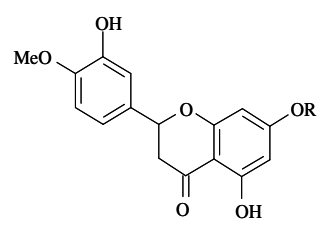

Hesperidin (R: rutinose)

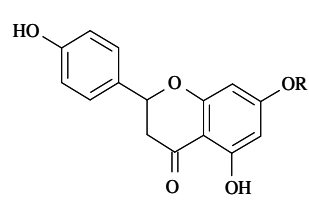

Naringin (R: neohesperidose)

most of polar and non-polar bioactive compounds such as flavonoids and pectic substances.

Almost all citrus flavonoids are contained in all parts of fruit except the juice. Figure 1 shows some kinds of flavonoids and chalcones.

Hesperidin and naringin are found in citrus genus, and their concentrations are much higher than many other flavonoids. These two flavanones have bioactivities such as hypolipidemic effect and apoptosis inducing effect in cancer cell [1]. It has been thought that hydroxylated chalcone, like phloretin, has anti-allergenic activity and induced apoptosis for human cancer cell [2,3]. Polymethoxyflavone tangeretin was also reported to have anti-proliferative and apoptotic effects on human cancer cell lines [4].

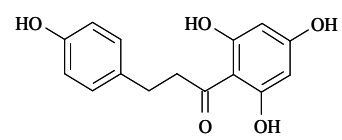

Phloretin

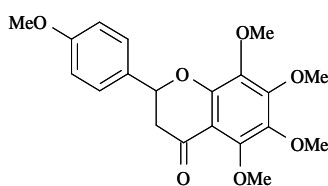

Tangeretin

Figure 1. Structural illustrations of some flavonoids of Yuzu peel

Extraction of flavonoids from plant materials has been accomplished by traditional extraction processes, such as solid-liquid extraction, using organic solvent such as methanol, ethanol, and acetone. [5] Even though high yield of target compounds can be obtained by organic solvent extraction, the use of organic solvent is not recommended because residual organic solvents have potential adverse effect on human health. Carbon dioxide 
and water are harmless, and present widely in nature. Therefore, subcritical water and $\mathrm{SC}-\mathrm{CO}_{2}$ have been applied individually to extract polar and non-polar compounds from various plant materials. Flavonoids are weakly polar, so it is difficult to effectively extract these compounds using SC- $\mathrm{CO}_{2}$ or subcritical water separately.

In this work, extraction and separation of various flavonoids from citrus junos peel using green solvents, e. g. $\mathrm{CO}_{2}$ and water, simultaneously were investigated by varying their operating conditions. $\mathrm{SC}-\mathrm{CO}_{2}$ is suitable for extracting weakly polar substances such as flavonoids by simply adding small amount of polar co-solvent such as ethanol and water.

\section{Materials and Methods}

\subsection{Materials and Chemicals}

Using yuzu flavedo as the starting material, the residue from supercritical $\mathrm{CO}_{2}$ extraction of essential oil at $60^{\circ} \mathrm{C}$ and $20 \mathrm{MPa}$ was supplied by ASCII Co. Ltd. (Fukuoka, Japan). The residue was freeze-dried, ground, and sieved. Flavedo with a particle size of $170-450 \mu \mathrm{m}$ was obtained as raw materials. Standard compounds of hesperidin, naringin, phloretin and tangeretin for use in HPLC analyses were purchased from Wako Pure Chem. Ind., Ltd (Osaka, Japan).

\subsection{Solvent Extraction}

A $0.1 \mathrm{~g}$ of material was extracted with $50 \%$ of methanol solution (methanol/DMSO=1/1, v/v) to investigate the total amount of flavonoids.

\subsection{HPLC Analysis of Flavonoid}

Flavonoid extracts were analyzed using a HPLC LC10AD gradient system, equipped with Diode Array Detector SDP-M10A. Inertsil ODS-3 column was used for separation at $35^{\circ} \mathrm{C}$. The mobile phase consisted of solvent A, $0.1 \%$ acetic acid in water, and solvent B, $0.1 \%$ acetic acid in acetonitrile (acetonitrile/water $=75 / 25, \mathrm{v} / \mathrm{v}$ ). The flow rate was $1.0 \mathrm{~mL} / \mathrm{min}$. Peaks were measured at a wavelength of $285 \mathrm{~nm}$ to quantify flavonoids. The gradient elution was as follows: time 0 min A-B (88:12); time 18 min A-B (78:22); time 28 min A-B (72:28); time $35 \mathrm{~min}$ A-B (62:38), time 48 min A-B (52:48), time 58 min A-B (0:100); time 70 min A-B (88:12). The flow rate was $1.0 \mathrm{~mL} / \mathrm{min}$. Peaks were measured at wavelength of $285 \mathrm{~nm}$ to quantify flavonoids.

\subsection{SC-CO $\mathrm{C}_{2}$ Extraction}

Figure 2 shows schematic diagram of $\mathrm{SC}-\mathrm{CO}_{2}$ extraction apparatus with an option of adding a co-solvent. The maximum working conditions of the apparatus are $450^{\circ} \mathrm{C}$ and $45 \mathrm{MPa}$. The pressure in the extractor was controlled by a back-pressure regulator (HBP-450; Akico Co., Ltd.). The extraction temperature was monitored by the thermocouples at the inlet and outlet of the extractor. In the flavonoids extraction, a $2.0 \mathrm{~g}$ portion of raw material was charged in the extractor $(10 \mathrm{~mL}) . \mathrm{SC}-\mathrm{CO}_{2}$ extraction of flavonoid was carried out at a pressure of 30 $\mathrm{MPa}$ and temperatures from 80 to $160^{\circ} \mathrm{C}$. The percent ratio of water in ethanol as a co-solvent was varied from 0 to $100 \%$. Flow rates of $\mathrm{CO}_{2}$ and co-solvent were also fixed at 3.0 and $0.2 \mathrm{~mL} / \mathrm{min}$, respectively.

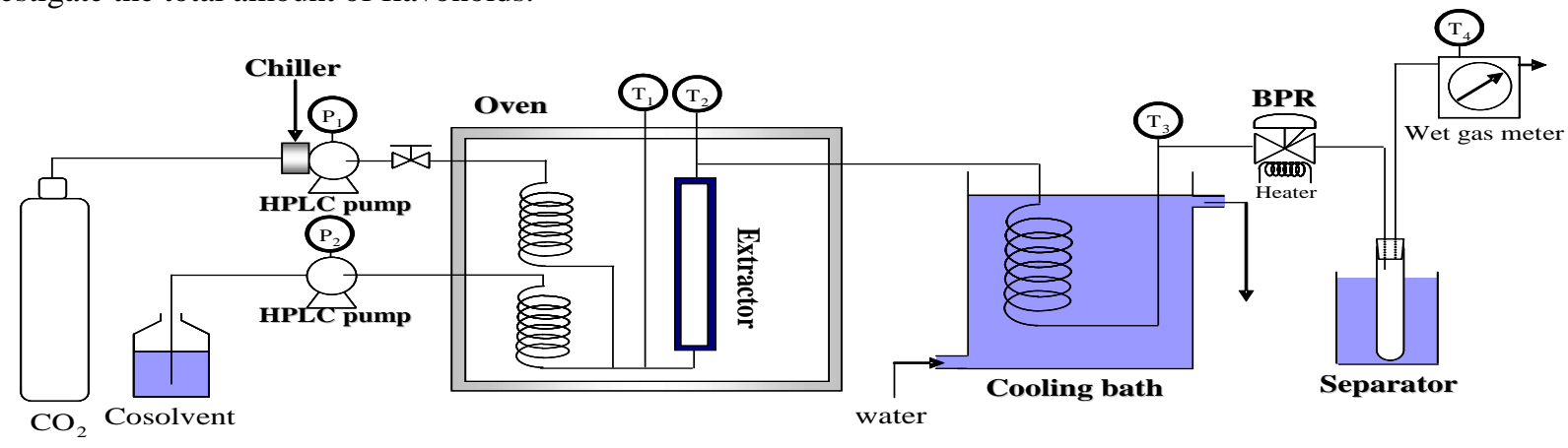

Figurer 2. Schematic diagram of SC- $\mathrm{CO}_{2}$ extraction apparatus with line for co-solvent

\subsection{Statistical Analysis}

All extraction experiments were duplicated. HPLC analysis of each fraction obtained in each run was carried out in duplicated. Analysis of variance (ANOVA) was carried out using Excel Statistics 2004 to analyze the effect of temperature and pressure on the total yield of extract and composition of essential oil extract. The significance level was stated 95\% with pvalue $<0.05$.

\section{Result and Disccusion}

\subsection{Identification and Quantitation}

The flavonoids in raw marteial were identified by comparison with commercial standards. Figure 3 shows HPLC chromatogram of flavonoids obtained by typical
SC- $\mathrm{CO}_{2}$ with co-solvent extract and conventional organic solvent extract.

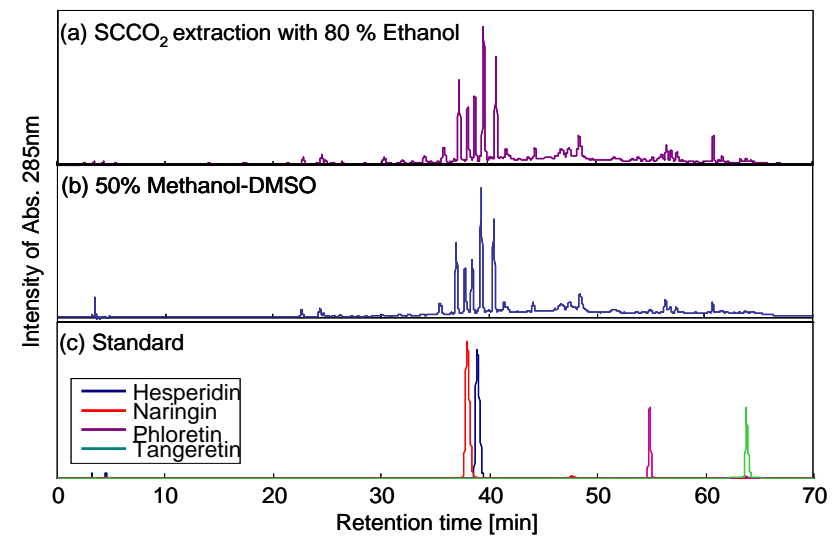

Figure 3. HPLC chromatograms of extract for $\mathrm{SC}-\mathrm{CO}_{2}$ with co-solvent (a) and conventional organic solvent (b) and standard substances (c) 
The closely matched spectra and retention times confirmed naringin, hesperidin, phloretin and tangeretin as important components in raw material, application of the peak purity software to the diode array data indicated no impurities present in any chromatographic peaks of interest.
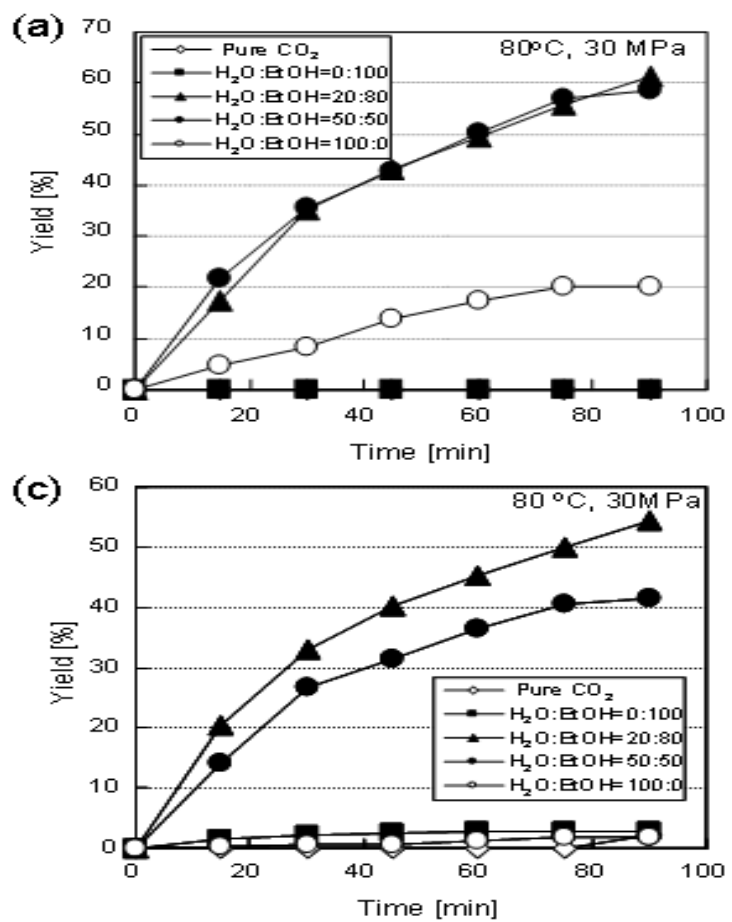

Figure 4. Effect of water content on SC- $\mathrm{CO}_{2}$ extraction of various flavonoids (a) Naringin, (b) Hesperidin, (c) Phloretin and (d) Tangeretin

Naringin and hesperidin were well extracted when water was added in ethanol. It was thought that naringin and hesperidin has higher polarity than other flavonoid due to having sugars in its structure. The yield of phloretin also increased with the addition of water. However, the increase of water content from 20 to $50 \%$ decreased the yield by about $15 \%$. The yield of tangeretin tended to increase with ethanol concentration. It was thought that the yield of tangeretin was related to low polarity derived
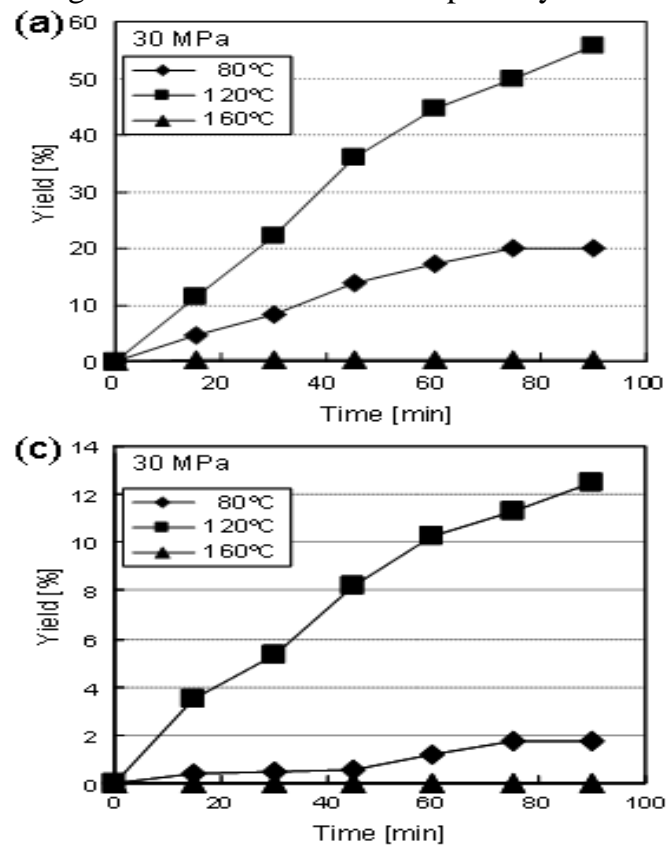

\subsection{Effect of Water Content on Flavonoid Extraction}

To study the effect of changing polarity of extracting solvent, $\mathrm{SC}-\mathrm{CO}_{2}$ extraction of flavonoid was carried out using co-solvent having various water contents in ethanol. Figure 4 shows the effect of water content on $\mathrm{SC}-\mathrm{CO}_{2}$ extraction of various flavonoids.
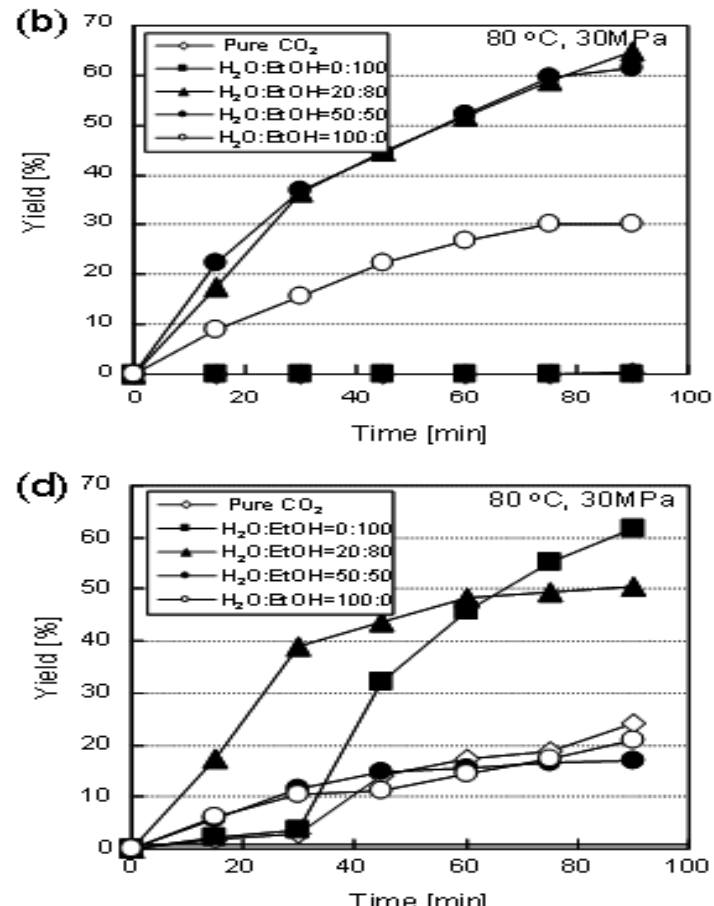

from having many methyl groups. These results showed selective extraction of flavonoids was possible by manipulating the ratio of water and ethanol.

\subsection{Effect of Temperature on Flavonoid Extraction}

Figure 5 shows the effect of temperature on extraction behavior of flavonoids using $\mathrm{SC}-\mathrm{CO}_{2}$ with only water as co-solvent.
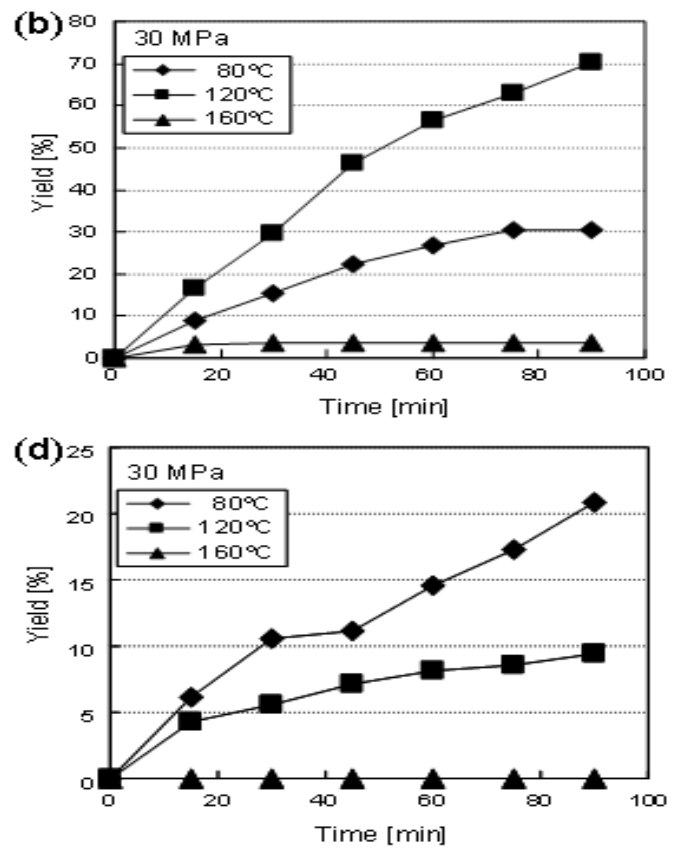

Figure 5. Effect of temperature on the yield of various flavonoids in SC-CO2 extraction with water (a) Naringin, (b) Hesperidin, (c) Phloretin, (d) Tangeretin 
The extraction of naringin and hesperidin was enhanced by temperature rise. It was indicated that flavonoid glycosides such as naringin and hesperidin extracted by using only green solvent. However extraction efficiency of flavonoids dramatically decreased due likely to partial degradation of flavonoid structure at the highest temperature of $160^{\circ} \mathrm{C}$.

\section{Conclusion}

Flavonoids such as naringin and hesperidin have glycoside group, thus water was a good solvent for extraction of these compounds. These results showed that it is possible to extract flavonoids selectively by changing the ratio of water and ethanol.

\section{Acknowledgement}

This work was supported by Kumamoto University Global COE Program "Global Initiative Center for Pulsed Power Engineering”.

\section{References}

[1] Kanno, S., Shouji, A., Asou, K. \& Ishikawa, M. Journal of Pharmaceutical Sciences, 92, (2003)166-170.

[2] Kanda, T., Akiyama, H., Yanagida, A., Tanabe, M., Goda, Y., Toyoda, M., Teshima, R., and Saito, Y., Biosci. Biotechnol. Biochem. 62(7), (1998), 1248-1289.

[3] Masuko Kobori”, Hiroshi Shinmoto, Tojiro Tsushida, Kazuki Shinohara, Phloretin-induced apoptosis in B 16 melanoma 4 A 5 cells by inhibition of glucose transmembrane transport, Cancer Letters 119 (1997) 207-212.

[4] Rodriguez, J., Yanez, J., Vincent, V., Alcaraz, M.,BenaventeGarcia, O., Castillo, J., Lorente, J., Lozano, J.A., Melanoma Res., 12, (2002), 99-107.

[5] Braddock, R. J., Handbook of Citrus By-products and Processing Technology, Willy Inter Science, New York, 1999. 